\section{Ocular and systemic factors associated with diabetes mellitus in the adult population in rural and urban China. The Beijing Eye Study}

$\mathrm{LX} \mathrm{u}^{1}, \mathrm{XW} \mathrm{Xie}^{1}, \mathrm{YX} \mathrm{Wang}^{1}$ and JB Jonas ${ }^{1,2}$
${ }^{1}$ Beijing Institute of Ophthalmology, Beijing Tongren Hospital, Capital Medical University, Beijing, China

${ }^{2}$ Department of Ophthalmology, Medical Faculty Mannheim of the Ruprecht-Karls-University of Heidelberg, Mannheim, Germany

Correspondence: JB Jonas or L Xu, Beijing Institute of Ophthalmology, 17 Hougou Lane, Chong Wen Men, 100005 Beijing, China. Tel: + 49621383 2652; Fax: + 496213833803 . E-mail: Jost.Jonas@ augen.ma.uni-heidelberg.de

Received: 24 October 2007 Accepted in revised form: 30 December 2007 Published online: 8 February 2008

Proprietary interest: None

\begin{abstract}
Purpose To assess ocular and systemic factors associated with diabetes mellitus in the adult population in rural and urban China.

Methods The Beijing Eye Study 2006, a population-based, cross-sectional cohort study, included 3251 subjects aged 45 years and more (participation rate: $73.2 \%$ ). Blood samples were available for $2960(91.0 \%)$ subjects. Diabetes mellitus was defined by a fasting plasma glucose concentration $\geq 7.0 \mathrm{mmol} / 1$ or by a self-reported history diagnosis of diabetes. Results Diabetes mellitus was found in 381 (12.9\%) subjects. In binary regression analysis, the presence of diabetes mellitus was significantly associated with body mass index, systolic blood pressure, triglyceride concentrations, intraocular pressure, cylindrical refractive dioptre, presence of arteriolar sheathing, rural vs urban region, lower best-corrected visual acuity, lower highdensity lipoprotein level, and lower diastolic blood pressure. It was not statistically associated with age, presence of cataract (nuclear, cortical, or subcapsular), size of the optic disc, neuroretinal rim, $\alpha$ zone and $\beta$ zone of peripapillary atrophy, retinal artery and vein diameters, arteriovenous nicking, focal or general narrowing, refractive error, prevalence of glaucoma, and early or late stage of agerelated macular degeneration.

Conclusions In a population-based setting, diabetes mellitus was not associated with optic disc, rim and peripapillary atrophy measurements, retinal vessel diameters, arteriovenous nicking, focal or general retinal artery narrowing, and prevalence of
\end{abstract}

age-related macular degeneration. Although diabetes mellitus was significantly correlated with higher intraocular pressure, it was not associated with glaucoma.

Eye (2009) 23, 676-682; doi:10.1038/sj.eye.6703104; published online 8 February 2008

Keywords: diabetes mellitus; optic disc; intraocular pressure; peripapillary atrophy; retinal vessel diameter; Beijing Eye Study

\section{Introduction}

Diabetes mellitus is, or is going to be, one the most important systemic diseases in all ethnic population groups through all groups of age with a pronounced impact on ocular function. ${ }^{1-5}$ It has been known for long that diabetic retinopathy, as the most important ocular complication of diabetes mellitus, is one of the leading causes of visual impairment worldwide. Associations of diabetes mellitus with ocular normative parameters, ocular abnormalities, and ophthalmic diseases other than diabetic retinopathy have usually not extensively been studied. It was, therefore, the purpose of the present population-based study to assess relationships between diabetes mellitus, ocular normative parameters, and the frequency of major ocular diseases with diabetes mellitus.

\section{Methods}

The Beijing Eye Study is a population-based cohort study in northern China that was performed in 2001 and included 4439 subjects 
aged 40 years and more. ${ }^{6,7}$ It was carried out in seven communities. Four communities were selected from the Haidian urban district of the northern part of central Beijing, and three communities were selected from the rural area of Yufa in the county of Daxing district, south of Beijing. In the rural areas, eye care services and a referral system to ophthalmologists were not available, and the cost for medical care was not covered by the government. In the urban areas, eye care was of a relatively high standard with some communities supplying free ophthalmic examinations and, in these areas, the cost of medical care was covered by the government. In 2006, the study was repeated, with 3251 subjects (1838 women) participating, corresponding to a response rate of $73.2 \%$. The study was divided into a rural part (1500 (46.1\%) subjects) and an urban part (1751 $(53.9 \%)$ subjects). Mean age was $60.4 \pm 10.0$ years (range, 45-89 years). The Medical Ethics Committee of the Beijing Tongren Hospital approved the study protocol and all participants gave informed consent.

All examinations were carried out in the communities, either in schoolhouses or in community houses. A detailed ophthalmic examination was carried out including measurement of uncorrected and bestcorrected visual acuity (Snellen charts), frequency doubling perimetry using the screening programme C-20-1 (Zeiss-Humphrey, Dublin, CA, USA), slit lampassisted optical coherent tomography of the anterior chamber morphology (Heidelberg Engineering, Heidelberg, Germany), ophthalmoscopy, and photography of the cornea, lens, and fundus. Intraocular pressure was measured using a non-contact pneumotonometer (CT-60 computerized tonometer, Topcon Ltd., Japan) by an experienced technician. Slit lamp examination was performed by an ophthalmologist, who had passed his ophthalmic training residency. During the slit lamp examination, the anterior chamber depth was assessed using van Herick's method. ${ }^{8}$ The pupil was dilated using tropicamide once or twice, until the pupil diameter was at least $6 \mathrm{~mm}$. Using the slit lamp, digital photographs of the cornea and lens were taken. Additionally, retro-illuminated photographs of the lens were taken. The degree of nuclear cataract was graded in six grades according to the lens photographs using the classifying scheme for cataract of the Age-Related Eye Disease Study. ${ }^{9}$ We combined standard photographs 6 and 7 into one grade, that is, 'grade 6 '. Grade ' 1 ' was no nuclear opacity in the lens, and grade ' 6 ' was very dense nuclear lens opacity. The degree of cortical lens opacification and posterior subcapsular lens opacification was graded using two photographs taken by retro-illumination with the Neitz CT-R camera (Neitz Instruments Co., Tokyo, Japan). Cortical and posterior subcapsular opacities appeared as darkly shaded areas on the white background on the computer screen. The percentage area of opacity was measured using a grid. ${ }^{10}$ Past history of eye diseases, eye trauma, diabetes mellitus, hypertension, and any ophthalmologic care the participant received was recorded. Additional information was obtained on the gross net family income, the level of education, and the profession. The level of education was differentiated in various degrees. 'Illiteracy' was defined as the inability to read any Chinese word. 'Half illiteracy' was present if the person could read a little of Chinese words, but could not get any useful information from the reading. The next degrees were 'Primary School', 'Middle School', and 'College Education', respectively, if a primary school, a middle school, or a college, respectively, had been attended.

Using the fundus photographs, the occurrence of retinal vein occlusions was assessed as described recently. ${ }^{11}$ In addition, the optic disc photographs were examined for the presence of glaucomatous optic nerve damage as described previously.,12 Glaucoma was defined by the glaucomatous appearance of the optic disc as the only criterion ('optic disc glaucoma'), or by additional perimetric defects in frequency doubling perimetry ('perimetric glaucoma'). ${ }^{7}$ The height of intraocular pressure was not a criterion for the diagnosis of 'optic disc glaucoma'. Intraocular pressure was not a criterion for the diagnosis. The optic disc photographs were additionally digitized, and the optic disc structures were measured by outlining the borders of the optic disc, optic cup, peripapillary scleral ring, and $\alpha$ zone and $\beta$ zone of peripapillary atrophy border on the computer screen. ${ }^{12}$

For the evaluation of the retinal vascular abnormalities, focal narrowing of arterioles, generalized narrowing of arterioles, sheathing of arterioles, and arteriovenous crossing abnormalities (arteriovenous nicking) were assessed. The colour optic disc photographs of one randomly selected eye of each subject were morphometrically examined using the assessment protocol of the Atherosclerosis Risk in Communities (ARIC) study. ${ }^{13,14}$ 'Definite focal narrowing' was present if the involved vessel had a diameter of at least $50 \mu \mathrm{m}$ or about one-third of the diameter of a major vein at the disc margin, and if the constricted area had a calibre of less than or equal to two thirds of the calibre of proximal and distal arterial segments. Retinal arterial narrowing was 'questionable', if the narrowing of the retinal artery or arteriole was less than $50 \mu \mathrm{m}$ (or less than $1 / 3$ of a major vein diameter at the disc margin) in calibre. Arteriolar sheathing was defined as an opacification of the arteriolar column. Arteriolar sheathing was graded as 'definite', if the arteriolar walls were partially opaque, that is, a ribbon of 
blood could still be seen with white lines on one or both sides, or if there was complete opacification of the arteriolar column. Arteriovenous crossing abnormalities (arteriovenous nicking) were graded as 'definite', if there was tapering or narrowing of the venous blood column on both sides of an arteriovenous crossing. If the venous blood column appears tapered on only one side of the crossing, and the appearance was not due to a normal vessel undulation, arteriovenous nicking was graded as 'questionable'. Any seeming decrease in venous calibre due to overlying retinal nerve fibres was discounted. The method has been described previously. ${ }^{14}$ Testing the inter-observer and intra-observer variability of the assessment, photographs of 50 randomly selected eyes were assessed by three trained ophthalmologists, independently of each other. One observer re-assessed the 50 photographs after about 2 weeks. The $\kappa$ values were $0.5-0.75$ for the intra-observation and 0.3-0.6 for inter-assessment of focal arteriolar narrowing, arteriovenous nicking, and arteriolar sheathing. For the assessment of generalized arteriolar narrowing, the kappa value was 0.3 for the intra-observer variation and 0.1 for the inter-observer examination. ${ }^{14}$

Fasting blood glucose concentration and the levels of triglycerides, low-density lipoproteins, high-density lipoproteins, and cholesterol were measured after obtaining blood samples. In addition, the systolic and diastolic blood pressure was determined. For study purposes, diabetes mellitus was defined as a fasting plasma glucose concentration $\geq 7.0 \mathrm{mmol} / 1$ or by a self-reported history diagnosis of diabetes.

Statistical analysis was performed by using a commercially available statistical software package (SPSS for Windows, version 14.0, SPSS, Chicago, IL, USA). Logistic regression was used to investigate the associations of the binary dependent variable 'presence of diabetes mellitus' with the continuous or categorical independent variables, such as age, gender, education level, and intraocular pressure. Odds ratios and 95\% confidence intervals (CI) were calculated to describe the influence of age, gender, area, and education degree to the prevalence of diabetes mellitus. Only one randomly selected eye per subject was considered for statistical analysis.

\section{Results}

Of the 3251 subjects included in the study, blood samples were available for $2960(91.0 \%)$ subjects. The mean age was $60.4 \pm 10.0$ years (median: 60 years; range: $45-89$ years), and the mean refractive error was $-0.20 \pm 2.08$ dioptres (median: 0.125 dioptres). For 291 (9.0\%) subjects, blood samples could not be obtained since the subjects refused the blood sampling. These non-participating subjects were compared with the study participants, who originated significantly more frequently from the urban region than from the rural region $(P<0.001)$, and did not vary in age $(P=0.95)$. Blood glucose concentrations higher than $\geq 7.0 \mathrm{mmol} / 1$ were detected in 227 subjects (prevalence rate (mean \pm s.e.): $7.67 \pm 0.49 \%$; 95\% CI: 6.71 , 8.63). From those subjects with a blood glucose concentration of $<7.0 \mathrm{mmol} / \mathrm{l}, 154$ subjects gave a selfreported diagnosis of diabetes mellitus. Combining the subjects with the self-reported diagnosis of diabetes mellitus and the hyperglycemic group, and defining them as diabetic revealed that 381 subjects (prevalence rate: $12.87 \pm 0.62 \%$; $95 \%$ CI: $11.66,14.08)$ were diabetic.

The diabetic study group compared with the nondiabetic study group in univariate statistical analysis showed significantly higher age, body mass index, systolic blood pressure, triglyceride and low-density lipoprotein concentrations, intraocular pressure, cylindrical refractive dioptre, lower high-density lipoprotein levels, lower diastolic blood pressure, and lower best-corrected visual acuity. In addition, the diabetic subjects came significantly more often from the urban region and had a higher prevalence of cataract (nuclear, cortical, or subcapsular) and arteriolar sheathing (Table 1). Diabetes mellitus was neither statistically associated with the size of the optic disc, neuroretinal rim, and $\alpha$ zone and $\beta$ zone of peripapillary atrophy, retinal artery and vein diameters, retinal microvascular abnormalities such as arteriovenous nicking, focal or general narrowing, refractive error, visual field defects, and the prevalence of glaucomatous optic nerve damage ('optic disc glaucoma' and 'perimetric glaucoma') as a whole nor differentiated into chronic open-angle glaucoma vs chronic angle-closure glaucoma, presence of retinal vein occlusions, presence of pterygium, and early or late stage of age-related macular degeneration (Table 2).

Performing a binary regression analysis, with the presence of diabetes mellitus as dependent variable and age, body mass index, systolic and diastolic blood pressure, level of triglycerides, low-density lipoproteins and high-density lipoproteins, intraocular pressure, visual field defects, presence of optic disc glaucoma, presence of pterygium, presence of cataract (nuclear, cortical, or subcapsular), and rural vs urban region as dependent parameters, revealed that diabetes mellitus was significantly associated with higher body mass index, systolic blood pressure, level of triglycerides, intraocular pressure, cylindrical refractive dioptre, presence of arteriolar sheathing, rural vs urban region, with lower best-corrected visual acuity, lower concentrations of high-density lipoproteins, and lower diastolic blood pressure. In the binary regression analysis, presence of diabetes mellitus was no longer 
Table 1 Differences in ocular and general parameters in the diabetic vs non-diabetic group of the Beijing Eye Study 2006

\begin{tabular}{|c|c|c|c|c|}
\hline $\mathrm{n}$ & $\begin{array}{c}\text { Non-diabetic group } \\
2579\end{array}$ & $\begin{array}{c}\text { Diabetic group } \\
381\end{array}$ & P-value & $95 \%$ confidence interval \\
\hline Age (years) & $59.7 \pm 10.0$ & $64.9 \pm 9.4$ & $<0.001$ & $-6.21,-4.16$ \\
\hline Body mass index & $25.4 \pm 3.7$ & $26.1 \pm 3.6$ & $<0.001$ & $-1.07,-0.28$ \\
\hline \multicolumn{5}{|l|}{ Blood pressure } \\
\hline Systolic & $134.9 \pm 20.8$ & $139.6 \pm 21.2$ & $<0.001$ & $-7.00,-2.50$ \\
\hline Diastolic & $82.3 \pm 11.1$ & $79.2 \pm 11.9$ & $<0.001$ & $1.91,4.32$ \\
\hline \multicolumn{5}{|l|}{ Serum concentrations } \\
\hline Cholesterol & $4.91 \pm 1.01$ & $4.94 \pm 1.09$ & 0.57 & $-0.14,0.08$ \\
\hline Triglycerides & $1.71 \pm 1.26$ & $2.10 \pm 1.48$ & $<0.001$ & $-0.54,-0.23$ \\
\hline Low-density lipoproteins & $2.86 \pm 0.86$ & $2.98 \pm 0.82$ & 0.015 & $-0.21,-0.02$ \\
\hline High-density lipoproteins & $1.51 \pm 0.35$ & $1.43 \pm 0.31$ & $<0.001$ & $0.06,0.13$ \\
\hline Intraocular pressure & $15.57 \pm 2.80$ & $16.14 \pm 2.96$ & $<0.001$ & $-0.87,-0.26$ \\
\hline Neuroretinal rim area & $1.63 \pm 0.35$ & $1.61 \pm 0.33$ & 0.63 & $-0.06,0.10$ \\
\hline$\alpha$ zone of peripapillary atrophy & $0.50 \pm 0.56$ & $0.52 \pm 0.57$ & 0.49 & $-0.09,0.04$ \\
\hline$\beta$ zone of peripapillary atrophy & $0.38 \pm 1.29$ & $0.36 \pm 0.94$ & 0.86 & $-0.13,0.16$ \\
\hline Central corneal thickness & $565.2 \pm 24.4$ & $558.1 \pm 20.5$ & 0.50 & $-14.6,28.8$ \\
\hline \multicolumn{5}{|l|}{ Retinal artery diameter } \\
\hline Temporal inferior & $0.109 \pm 0.017$ & $0.110 \pm 0.015$ & 0.48 & $-0.003,0.002$ \\
\hline Temporal superior & $0.103 \pm 0.016$ & $0.103 \pm 0.013$ & 0.99 & $-0.002,0.002$ \\
\hline Nasal superior & $0.088 \pm 0.015$ & $0.090 \pm 0.014$ & 0.10 & $-0.004,0.001$ \\
\hline Nasal inferior & $0.086 \pm 0.014$ & $0.088 \pm 0.013$ & 0.13 & $-0.004,0.001$ \\
\hline \multicolumn{5}{|l|}{ Retinal vein diameter } \\
\hline Temporal inferior & $0.153 \pm 0.023$ & $0.152 \pm 0.022$ & 0.48 & $-0.002,0.005$ \\
\hline Temporal superior & $0.145 \pm 0.023$ & $0.145 \pm 0.021$ & 0.95 & $-0.003,0.004$ \\
\hline Nasal superior & $0.110 \pm 0.019$ & $0.110 \pm 0.017$ & 0.86 & $-0.003,0.003$ \\
\hline Nasal inferior & $0.109 \pm 0.033$ & $0.106 \pm 0.020$ & 0.24 & $-0.002,0.008$ \\
\hline Visual field defects & $0.81 \pm 3.84$ & $1.27 \pm 4.39$ & 0.06 & $-0.93,0.01$ \\
\hline Best-corrected visual acuity & $0.96 \pm 0.21$ & $0.86 \pm 0.25$ & $<0.001$ & $0.07,0.12$ \\
\hline Refractive error & $-0.20 \pm 2.10$ & $-0.21 \pm 1.96$ & 0.90 & $-0.21,0.24$ \\
\hline Cylindrical refractive error & $0.64 \pm 0.66$ & $0.88 \pm 0.76$ & $<0.001$ & $-0.33,-0.16$ \\
\hline
\end{tabular}

$P$-value: statistical significance of differences between the two study groups (univariate analysis).

associated with age $(P=0.49)$, low-density lipoproteins $(P=0.67)$, and presence of cataract (nuclear, cortical, or subcapsular ( $P=0.21,0.46,0.81$, respectively)) (Table 3 ).

Correlating the level of fasting glucose concentration showed significant associations with higher age, systolic blood pressure, triglycerides and low-density lipoprotein concentrations, and intraocular pressure, with lower diastolic blood pressure, and lower levels of high-density lipoproteins. In this multivariate analysis, body mass index $(P=0.09)$, age $(P=0.15)$, and rural $v$ s urban region $(P=0.46)$ were not significantly associated with the fasting plasma concentration of glucose (Table 4).

\section{Discussion}

In the present population-based study, performed in a rural region and an urban region of greater Beijing, diabetes mellitus was neither associated with optic disc, neuroretinal rim and peripapillary atrophy measurements, retinal vessel diameters, arteriovenous nicking, focal or general narrowing, and prevalence of age-related macular degeneration, retinal vein occlusions, and glaucoma as a whole nor differentiated into chronic open-angle glaucoma vs chronic angleclosure glaucoma. As expected from numerous previous studies, presence of diabetes mellitus was correlated with the systemic parameters body mass index, systolic blood pressure, triglyceride concentration, and lower high-density lipoprotein level.

Interestingly, the presence of diabetes mellitus as well as the level of fasting plasma glucose concentration were correlated with intraocular pressure, although the neuroretinal rim was not smaller, nor was the beta zone of peripapillary atrophy larger, and the prevalence of glaucomatous optic nerve damage also did not increase. Since elevated intraocular pressure is the major risk factor for the development of glaucomatous optic nerve damage, it has remained unclear why in the present population-based study, the association between elevated intraocular pressure and diabetes mellitus did 
Table 2 Differences in the prevalence of ocular diseases in the diabetic vs non-diabetic group of the Beijing Eye Study 2006

\begin{tabular}{|c|c|c|c|c|c|}
\hline & $\begin{array}{l}\text { Non-diabetic } \\
\quad \text { group }\end{array}$ & $\begin{array}{l}\text { Diabetic } \\
\text { group }\end{array}$ & P-value & $\begin{array}{l}\text { Odds } \\
\text { ratio }\end{array}$ & $\begin{array}{l}95 \% \text { confidence } \\
\text { interval }\end{array}$ \\
\hline Rural vs urban region & $49.5 / 50.5$ & $34.6 / 65.4$ & $<0.001$ & 1.85 & $1.48,2.32$ \\
\hline \multicolumn{6}{|l|}{ Lens } \\
\hline Nuclear cataract & $4.3 \%$ & $7.8 \%$ & 0.004 & 1.88 & $1.22,2.90$ \\
\hline Cortical cataract & $8.1 \%$ & $15.9 \%$ & $<0.001$ & 2.16 & $1.57,2.97$ \\
\hline Subcapsular cataract & $3.6 \%$ & $6.5 \%$ & 0.009 & 1.87 & $1.16,3.00$ \\
\hline \multicolumn{6}{|l|}{ Retinal microvascular abnormalities } \\
\hline \multicolumn{6}{|l|}{ Focal narrowing } \\
\hline Definite & $6.3 \%$ & $5.2 \%$ & 0.40 & 0.81 & $0.50,1.32$ \\
\hline Definite + questionable & $13.5 \%$ & $10.9 \%$ & 0.17 & 0.78 & $0.55,1.11$ \\
\hline Generalized narrowing & $3.9 \%$ & $4.4 \%$ & 0.70 & 1.11 & $0.65,1.91$ \\
\hline \multicolumn{6}{|l|}{ Arteriovenous nicking } \\
\hline Definite & $7.0 \%$ & $6.8 \%$ & 0.87 & 0.97 & $0.63,1.49$ \\
\hline Definite + questionable & $8.5 \%$ & $8.4 \%$ & 0.99 & 0.87 & $0.67,1.48$ \\
\hline \multicolumn{6}{|l|}{ Arteriolar sheathing } \\
\hline Definite & $4.3 \%$ & $8.7 \%$ & $<0.001$ & 2.13 & $1.41,3.21$ \\
\hline Definite + questionable & $5.0 \%$ & $9.8 \%$ & $<0.001$ & 2.06 & $1.40,3.04$ \\
\hline 'Optic disc glaucoma' & $3.1 \%$ & $4.5 \%$ & 0.15 & 1.48 & $0.87,2.53$ \\
\hline 'Perimetric glaucoma' & $1.9 \%$ & $2.4 \%$ & 0.54 & 1.25 & $0.61,2.56$ \\
\hline $\begin{array}{l}\text { Chronic open-angle glaucoma/chronic angle-closure glaucoma } \\
\text { ('optic disc glaucoma') }\end{array}$ & $73.4 / 26.6$ & $94.1 / 5.9$ & 0.11 & 0.17 & $0.02,1.38$ \\
\hline \multicolumn{6}{|l|}{ Age-related macular degeneration } \\
\hline Early stage & $2.4 \%$ & $3.1 \%$ & 0.41 & 1.30 & $0.69,2.43$ \\
\hline Late stage & $0.2 \%$ & $0.3 \%$ & 0.91 & 1.13 & $0.14,9.40$ \\
\hline Pterygium & $2.8 \%$ & $1.6 \%$ & 0.18 & 0.57 & $0.24,1.31$ \\
\hline Retinal vein occlusions & $1.1 \%$ & $1.0 \%$ & 0.95 & 0.97 & $0.34,2.77$ \\
\hline
\end{tabular}

$P$-value: statistical significance of differences between the two study groups (univariate analysis; $\chi^{2}$ test).

Table 3 Binary logistic regression analysis between presence of diabetes mellitus and ocular and systemic parameters, which were significantly associated with diabetes mellitus in univariate analysis in the Beijing Eye Study

\begin{tabular}{|c|c|c|c|}
\hline Parameter & P-value & Odds ratio & $95 \%$ confidence interval \\
\hline Body mass index & 0.004 & 1.056 & $1.02,1.10$ \\
\hline Systolic blood pressure & $<0.001$ & 1.020 & $1.01,1.03$ \\
\hline Diastolic blood pressure & $<0.001$ & 0.952 & $0.94,0.97$ \\
\hline Triglycerides & 0.001 & 1.151 & $1.06,1.25$ \\
\hline High-density lipoproteins & 0.004 & 0.565 & $0.38,0.83$ \\
\hline Intraocular pressure & 0.027 & 1.051 & $1.01,1.10$ \\
\hline Rural vs urban region & $<0.001$ & 1.858 & $1.40,2.47$ \\
\hline Best-corrected visual acuity & 0.002 & 0.347 & $0.18,0.68$ \\
\hline Cylindrical refractive dioptre & 0.021 & 1.252 & $1.03,1.52$ \\
\hline Arteriolar sheathing & $<0.001$ & 2.409 & $1.55,3.74$ \\
\hline
\end{tabular}

not lead to an association between diabetes mellitus and glaucoma. It has remained elusive whether the increased blood pressure in the diabetic subjects compensated for the increased intraocular pressure with respect to an intraocular pressure-induced damage to the optic nerve. It may even be more speculative, whether the increased blood pressure in the diabetic subjects increased the cerebrospinal fluid pressure, which may act as translamina cribrosa counter pressure against the intraocular pressure. ${ }^{15-17}$
Although diabetes mellitus is a microvascular disease leading to tissue ischaemia, the diabetic subgroup did not vary significantly in size of neuroretinal rim from the non-diabetic subgroup. It is paralleled by hospital-based studies, in which a loss of neuroretinal rim was observed in glaucoma and giant-cell arteritis-associated anterior ischaemic optic neuropathy, while in other ischaemic retinal or optic nerve diseases, such as non-arteritic anterior ischaemic optic neuropathy, the neuroretinal rim did not change in size. ${ }^{17,18}$ In a similar manner, $\alpha$ zone 
Table 4 Multiple regression analysis between the plasma concentration of fasting glucose and ocular and systemic parameters, which were significantly associated with diabetes mellitus in univariate analysis in the Beijing Eye Study

\begin{tabular}{lcccc}
\hline Parameter & P-value & Steepness of the regression line & Correlation coefficient & 95\% confidence interval \\
\hline Body mass index & 0.088 (n.s.) & & & $-0.005,0.033$ \\
Systolic blood pressure & $<0.001$ & 0.011 & 0.139 & $0.008,0.015$ \\
Diastolic blood pressure & 0.006 & -0.009 & -0.061 & $-0.016,-0.003$ \\
Triglycerides & 0.001 & 0.085 & 0.065 & $0.036,0.133$ \\
Low-density lipoproteins & $<0.001$ & 0.185 & 0.094 & $0.113,0.257$ \\
High-density lipoproteins & 0.026 & -0.199 & -0.041 & $-0.375,-0.023$ \\
Intraocular pressure & $<0.001$ & 0.049 & 0.083 & $0.028,0.071$ \\
\hline
\end{tabular}

and $\beta$ zone of peripapillary atrophy did not differ significantly between the diabetic subjects and the non-diabetic subjects, again confirming previous hospital-based studies that both zones do not change in vascular diseases in contrast to glaucoma. ${ }^{17}$ It remains unclear whether the lack of an association between size of peripapillary atrophy and neuroretinal rim on one side and diabetes mellitus as microvascular disease on the other side point against a predominantly vascular pathogenesis of glaucomatous optic nerve damage.

Glaucoma and age-related macular degeneration neither in the early stage nor in the late stage were associated with diabetes mellitus (Table 2). Mostly confirming previous studies, this may suggest that the pathogenesis of age-related macular degeneration may not be due to a similar microvascular mechanism as in diabetes mellitus. ${ }^{19-26}$ In the binary analysis, the presence of diabetes mellitus was significantly associated with the body mass index. The association with body mass index was, however, statistically weaker than the associations between diabetes mellitus and blood pressure, levels of triglycerides, rural vs urban region, and the presence of arteriolar sheathing (Table 3). Interestingly, the fasting plasma glucose concentration was not significantly associated with the body mass index. Future studies may, therefore, address the question whether in Asians the association between diabetes mellitus and obesity may be less pronounced than that in Western countries.

There are limitations of the present study. A major concern in any prevalence study is non-participation. The Beijing Study 2006 had a reasonable response rate of $73 \%$, however, differences between participants and non-participants can lead to a selection artefact and may have produced an underestimate of the prevalence rates of diabetes mellitus and ocular diseases, such as glaucoma and age-related macular degeneration. Another limitation of the study is the definition of glaucoma, as used in the present study. An oral glucose tolerance test was not performed which may have served to further strengthen the diagnosis of diabetes mellitus.

In conclusion, in the Beijing Eye Study, diabetes mellitus was not associated with optic disc, rim and peripapillary atrophy measurements, retinal vessel diameters and arteriovenous nicking, focal or general narrowing, and prevalence of age-related macular degeneration. Although diabetes mellitus was significantly correlated with higher intraocular pressure, it was not associated with glaucoma. The statistically relatively weak association between diabetes mellitus and the body mass index may warrant further studies on the association between diabetes mellitus and obesity in China.

\section{Acknowledgements}

Supported by the National Key Laboratory Fund, Beijing, China.

\section{References}

1 Wild S, Roglic G, Green A, Sicree R, King H. Global prevalence of diabetes, estimates for the year 2000 and projections for 2030. Diabetes Care 2004; 27: 1047-1053.

2 Pan XR, Yang WY, Li GW, Liu J. Prevalence of diabetes and its risk factors in China, 1994: National Diabetes Prevention and Control Cooperative Group. Diabetes Care 1997; 20: 1664-1669.

3 King H, Aubert RE, Herman WH. Global burden of diabetes, 1995-2025: prevalence, numerical estimates, and projections. Diabetes Care 1998; 21: 1414-1431.

4 Jia WP, Pang C, Chen L, Bao YQ, Lu JX, Lu HJ et al. Epidemiological characteristics of diabetes mellitus and impaired glucose regulation in a Chinese adult population: the Shanghai Diabetes Studies, a cross-sectional 3-year follow-up study in Shanghai urban communities. Diabetologia 2007; 50: 286-292.

5 Gu D, Reynolds K, Duan X, Xin X, Chen J, Wu X et al. Prevalence of diabetes and impaired fasting glucose in the Chinese adult population: International Collaborative Study of Cardiovascular Disease in Asia (InterASIA). Diabetologia 2003; 46: 1190-1198.

6 Xu L, Wang Y, Li Y, Li J, Wang Y, Cui T et al. Causes of blindness and visual impairment in an urban and rural area in Beijing: the Beijing Eye Study. Ophthalmology 2006; 113: 1141.e1-1141.e3.

7 Xu L, Wang Y, Wang S, Wang Y, Jonas JB. High myopia and glaucoma susceptibility. The Beijing Eye Study. Ophthalmology 2007; 114: 216-220. 
8 Van Herick W, Shafer RN, Schwartz A. Estimation of width of angle of anterior chamber. Incidence and significance of the narrow angle. Am J Ophthalmol 1969; 68: 626-629.

9 Age-Related Eye Disease Study Research Group. The AgeRelated Eye Disease Study (AREDS) system for classifying cataracts from photographs: AREDS report no. 4. Am J Ophthalmol 2001; 131: 167-175.

10 Xu L, Cui T, Zhang S, Sun B, Zheng Y, Hu A et al. Prevalence and risk factors of lens opacities in urban and rural Chinese in Beijing. Ophthalmology 2006; 113: 747-755.

11 Liu W, Xu L, Jonas JB. Retinal vein occlusions in adult Chinese. Ophthalmology 2007; 114: 1795-1796.

12 Jonas JB, Budde WM, Panda-Jonas S. Ophthalmoscopic evaluation of the optic nerve head. Surv Ophthalmol 1999; 43: 293-320.

13 Hubbard LD, Brothers RJ, King WN, Clegg JX, Klein R, Cooper LS et al. Methods for evaluation of retinal microvascular abnormalities associated with hypertension/ sclerosis in the atherosclerosis risk in communities study. Ophthalmology 1999; 106: 2269-2280.

14 Wang S, Xu L, Jonas JB, Wang YS, Wang Y, Yang $\mathrm{H}$ et al. Retinal vascular abnormalities in adult Chinese in rural and urban Beijing. The Beijing Eye Study. Ophthalmology 2006; 113: 1752-1757.

15 Jonas JB, Mardin CY, Schlötzer-Schrehardt U, Naumann $\mathrm{GOH}$. Morphometry of the human lamina cribrosa surface. Invest Ophthalmol Vis Sci 1991; 32: 401-540.

16 Morgan WH, Yu DY, Alder VA, Cringle SJ, Cooper RL, House $\mathrm{PH}$ et al. The correlation between cerebrospinal fluid pressure and retrolaminar tissue pressure. Invest Ophthalmol Vis Sci 1998; 39: 1419-1428.

17 Jonas JB, Xu L. Optic disc morphology in eyes after nonarteritic anterior ischemic optic neuropathy. Invest Ophthalmol Vis Sci 1993; 34: 2260-2265.
18 Hayreh SS, Jonas JB. Optic disc morphology after arteritic anterior ischemic optic neuropathy. Ophthalmology 2001; 108: 1586-1594.

19 Klein R, Klein BE, Franke T. The relationship of cardiovascular disease and its risk factors to age-related maculopathy. The Beaver Dam Eye Study. Ophthalmology 1993; 100: 406-414.

20 Age-Related Eye Disease Study Research Group. Risk factors associated with age-related macular degeneration. A case-control study in the age-related eye disease study: AgeRelated Eye Disease Study Report Number 3. Ophthalmology 2000; 107: 2224-2232.

21 Hyman L, Schachat AP, He Q, Leske MC. Hypertension, cardiovascular disease, and age-related macular degeneration. Age-Related Macular Degeneration Risk Factors Study Group. Arch Ophthalmol 2000; 118: 351-358.

22 Smith W, Assink J, Klein R, Mitchell P, Klaver CC, Klein BE et al. Risk factors for age-related macular degeneration: Pooled findings from three continents. Ophthalmology 2001; 108: 697-704.

23 van Leeuwen R, Ikram MK, Vingerling JR, Witteman JC, Hofman A, de Jong PT. Blood pressure, atherosclerosis, and the incidence of age-related maculopathy: the Rotterdam Study. Invest Ophthalmol Vis Sci 2003; 44: 3771-3777.

24 Seddon JM, Cote J, Davis N, Rosner B. Progression of agerelated macular degeneration: association with body mass index, waist circumference, and waist-hip ratio. Arch Ophthalmol 2003; 121: 785-792.

25 Buch H, Vinding T, la Cour M, Jensen GB, Prause JU, Nielsen NV. Risk factors for age-related maculopathy in a 14-year follow-up study: the Copenhagen City Eye Study. Acta Ophthalmol Scand 2005; 83: 409-418.

26 Tan JS, Mitchell P, Smith W, Wang JJ. Cardiovascular risk factors and the long-term incidence of age-related macular degeneration the Blue Mountains Eye Study. Ophthalmology 2007; 114: 1143-1150. 